\section{Manual Active Cannula Deployment: Experimental Accuracy Evaluation in Free Space}

\section{Marlena S. Clark, Jessica Burgner, Philip J. Swaney, and Robert J. Webster III}

Department of Mechanical Engineering

Vanderbilt University

\section{Background}

Liver tumor ablation requires the ability to accurately target multiple sites with an ablator tip using an insertion device. A minimally invasive approach using straight needles usually requires multiple insertions which damage the liver capsule and cause bleeding. We propose a multi-tube steerable cannula to enable access to multiple internal points from a single liver capsule entry point. The cannula is made of concentric tubes of superelastic Nitinol which can translate and rotate with respect to one another in order to drive the tip to a desired target, through a curved path.

Deployment of the cannula from an insertion unit can be entirely automated [1], teloperated [2], or done manually [3]. For a first assessment towards human clinical trials, we built a manual insertion unit that is completely sterilizable. In this paper we evaluate the achievable accuracy of this manual actuation unit for deploying the ablator tip to a desired Cartesian position in free space.

\section{Methods}

The manual insertion unit assessed here uses two concentric tubes. Each tube has a corresponding carrier which can move independently along a lead screw by rotation of control knobs. A worm gear is used to axially rotate each tube on its respective carrier, thereby determining the trajectory of the ablator tip as the tubes are inserted.

The experimental setup is shown in Fig 1. The manual insertion unit was rigidly fixed to a bench and deployed the cannula into an open container lined with corkboard. An optical tracking device (MicronTracker H3-60, Claron Technology, USA) was utilized to determine the position of the end effector using a point probe. The probe tip was tracked in reference to a frame attached to the base of the insertion unit.

To deploy the cannula to a Cartesian position measured with the point probe, the transformation matrix between the reference frame and the cannula frame is required. Point based registration using 25 points was performed prior to the experiment to obtain this matrix. During registration, the tubes curvatures and the inner tube curved length were concurrently estimated.

For evaluation of the accuracy of the manual insertion unit, 26 target points were selected by placing rods of varying length into the corkboard. In doing this, the positions could be visualized simultaneously, ensuring efficient use of the obtainable workspace. Each of these target positions was measured three times using a tracked point probe and averaged. For the targeting experiment each target position was transformed into the cannula frame using the registration transformation matrix. Inverse kinematics was used to determine the corresponding set of actuator values for the desired end effector position. For each set, the cannula was driven forward and its tip position was measured using the point probe. The end effector locations were recorded as an average of three trials. Between each trial, the cannula was moved back to its home position. The accuracy was then determined by calculating the Cartesian error between the desired and actual tip positions.

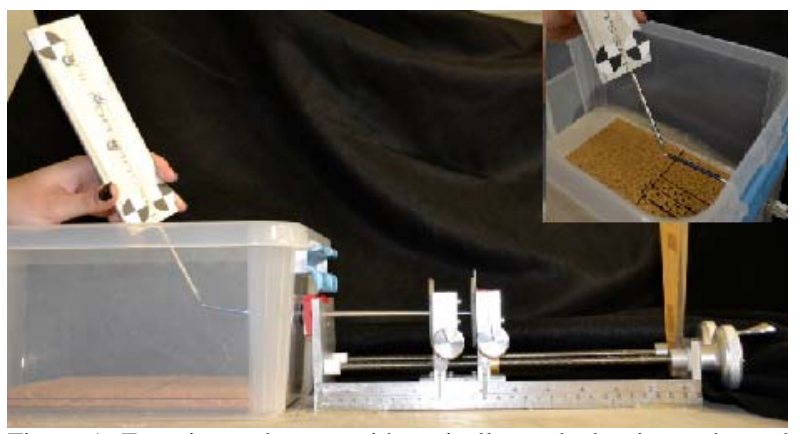

Figure 1: Experimental setup with optically tracked point probe and active cannula deployed with manual insertion unit.

\section{Results}

The root mean square error of registration was $1.18 \mathrm{~mm}$. The accuracy evaluation of the manual insertion unit resulted in a mean Cartesian error of $2.66 \mathrm{~mm}$ with a standard deviation of $1.63 \mathrm{~mm}$.

\section{Interpretation}

This study was performed to determine the overall accuracy of a manual insertion unit to target a desired point in free space. The resulting mean error is $2.66 \mathrm{~mm}$, which is likely to be clinically sufficient for targeting liver tumors. In addition to liver ablation, cannulas like ours appear able to deliver any kind of needle-based therapy or sample collection (e.g. drug injection, brachytherapy, biopsy, laser fiber delivery, etc). It is also possible to use robotic actuation to enable telesurgery with miniature grippers [2].

It is likely that manual actuation will only be sufficient for needle-like operation, particularly in initial clinical studies. It is of future interest to evaluate the accuracy of our manual insertion unit in ex-vivo and in-vivo human tissue using image-guidance from various medical imaging modalities.

\section{References}

[1] Burdette, E.C., Rucker, D.C., Prakash, P., Diederich, C.J., Croom, C.M., Clarke, C., Stolka, P.J., Juang, T., Boctor, E.M., and Webster III, R.J., 2010, "The ACUSITT Ultrasonic Ablator: The First Steerable Needle with an Integrated Interventional Tool,” SPIE Medical Imaging, 2010.

[2] Burgner, J., Swaney, P.J., Rucker, D.C., Gilbert, H.B., Nill, S.T., Russel III, P.T., Weaver, K. D., and Webster III, R.J., 2011, “A Bimanual Teloperated System for Endonasal Skull Base Surgery,” IEEE IROS, 2011.

[3] Bruns T.L., Tucker, J. M., Rucker, D. C., Swaney, P.J., Boctor, E.M., Burdette, E.C., Burgner, J., and Webster III, R.J., 2011, "Design of an Autoclavable Active Cannula Deployment Device,” ASME DMD, 2011. 